\title{
Design and Analysis of a Multiple-Input Receiver for Mimo Wireless Applications
}

\author{
Constantinos Votis, Panos Kostarakis \\ Physics Department, University of Ioannina, Panepistimioupolis, Ioannina, Greece \\ E-mail:kvotis@grads.uoi.gr,kostarakis@uoi.gr \\ Received April 21, 2010; revised May 28, 2010; accepted July 1, 2010
}

\begin{abstract}
In this article, we present multiple-input receiver architecture for (Multiple-Input Multiple-Output) MIMO wireless communication applications. The proposed implementation is provided by a defined number of identical receiver units that are fed by a RF modulated signal on specific carrier frequency, power strength and initial phase. These units carry out the corresponding amplification, filtering and demodulation procedures. Details on design and implementation of this Printed-Circuit-Board are introduced and further discussed. Experimental results are also presented, allowing the validation of investigation on the performance of the current receiver architecture. Besides, these measurements indicate that the proposed device, combining with a suitable antenna array, provides a versatile receiver platform for baseband signal processing. The incoming RF modulated signals have frequencies on the range of $2.4 \mathrm{GHz}$, several phases, magnitudes and modulation modes. From these, it seems that the proposed receiver implementation supports MIMO communication and multiple port channel characterization applications at $2.4 \mathrm{GHz}$ ISM (Industrial, Scientific and Medical) band.
\end{abstract}

Keywords: Channel Sounder, MIMO Systems, Baseband Processing

\section{Introduction}

Modern wireless communication systems continue to push for wider bandwidth capabilities, higher data rates and better quality of services. Scientific and engineering community provides a number of novel techniques and methods to meet these requirements. One of them is called Multiple-Input Multiple-Output (MIMO) architecture that could exploit the capacity of a wireless communication channel [1-3]. Using multiple antenna elements on both the transmitter and receiver ends offers significant capacity enhancement on radio propagation applications. In order to achieve this benefit, appropriate design aspects on such systems have to be taken into account. It is obvious that a receiver device with multiple input ports is mainly required. Furthermore, appropriate synchronization and data acquisition procedures have to be supported by this device in order to collect and record the data transmission streams from each sub-channel at any scattering radio propagation environment.

The efficiency of such systems depends on several performance and channel parameters. One of them is referred to the profound knowledge of the time-variant radio channel in various indoor or outdoor environments.
Devices that could provide knowledge of the wireless channel status are referred as channel sounding systems. Furthermore, several multiplexing techniques are applied to these systems for channel estimation purposes. Time, frequency, code division multiplexing and hybrid methods are mainly used in these applications [4,5]. Generally, these devices improve MIMO system performance and offer crucial assumptions that provide a resource for channel model developments.

In addition, the hardware is crucial for the performance of such MIMO systems. Resolution accuracy and capability are dominated by the corresponding strategy adopted for the channel sounder and communication applications. In particular, the choice of the receiver architecture indicates the method of channel acquisition and estimation, as well as the efficiency of the MIMO communication system. More precisely, fully switched, semiswitched and parallel transmission are the main techniques that supports channel characterization applications, using one or a combination of the multiplexing methods (TDM, FDM, CDM), each with different advantages and drawbacks.

These methods also support MIMO communication systems, providing transmit and receive diversity and 
channel capacity enhancements. Increment on data rates, decrement on bit error rates, independent sub-channels existence and quite excellent quality of services are the main advantages that are provided by them.

In order to design and construct a RF platform for MIMO channel sounder and communication applications, we propose this multiple-input receiver architecture. Existing experimental measurements set-ups are usually not able to probe a number of parallel streams, simultaneously. This feature is crucial for MIMO channel characterization and communication applications. In any case, the proposed receiver device corresponds to an experimental testbed that enables multiple channel applications, simultaneously. Due to this fact, an antenna array configuration is required for feeding the inputs of the proposed receiver implementation. Design and implementation aspects of this device and its complementary circuitry, including radio-frequency amplifying and down- conversion as well as calibration procedure are included in Section 2 of the current paper. In Section 3, the multi-channel receiver performance is also simulated on MIMO applications and further baseband signal processing techniques are also included, as well as the corresponding results are discussed; the paper concludes in Section 4 .

\section{Multiple-Input Receiver Design and Implementation}

The proposed implementation employs broadband quadrature demodulator architecture and comprises of several identical units, which operate synchronously and are fed by a matched multiple-element antenna array. A schematic block diagram of this receiver architecture is depicted in Figure 1. Besides, Figure 2 shows the corre-

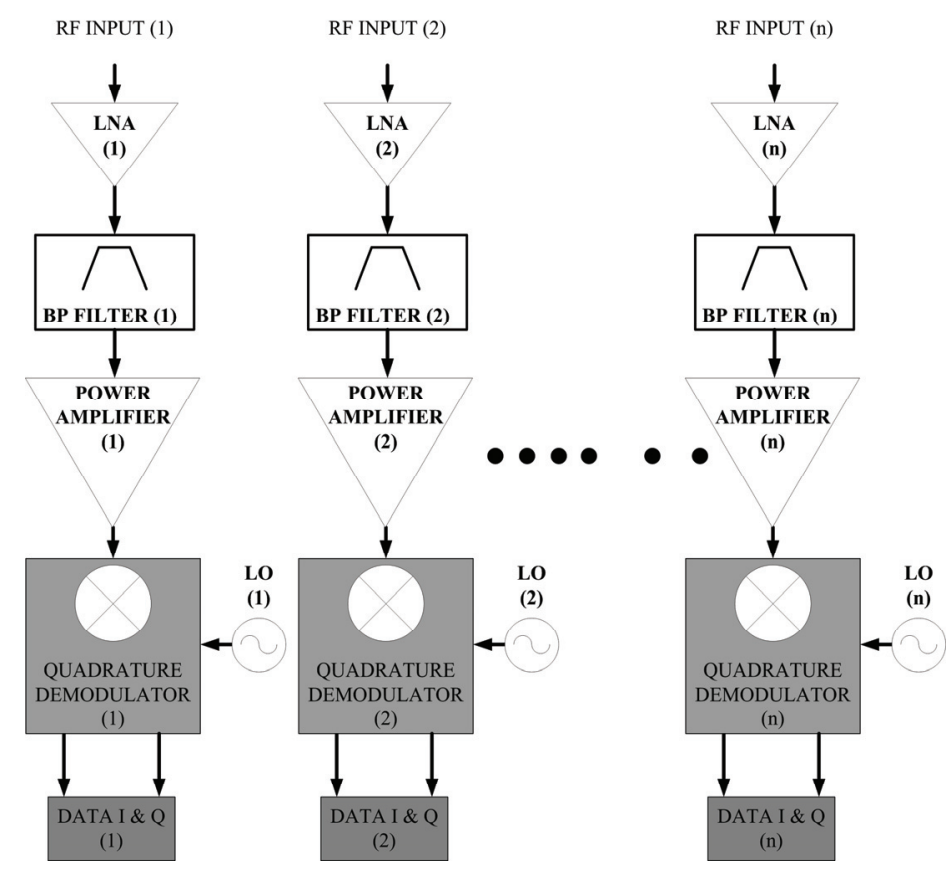

Figure 1. Generic receiver architecture.

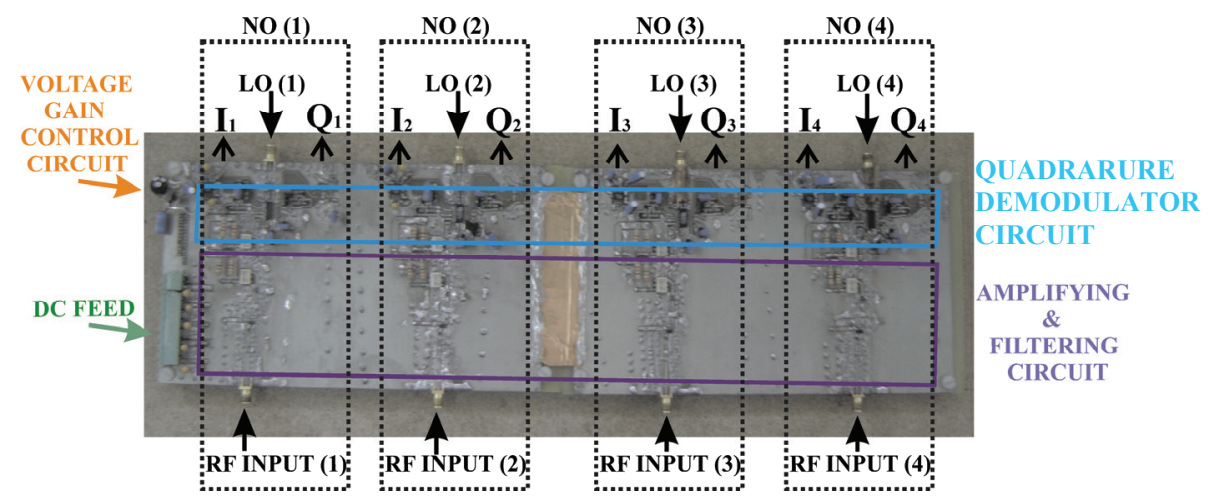

Figure 2. Receiver hardware implementation. 
sponding Printed-Circuit-Board (PCB).

In Figures $\mathbf{1}$ and 2, it is obvious that the first stage, at each of the receiver units, is represented by an appropriate amplifying and filtering circuitry. This provides a quite great power gain, increasing the received signal strength and eliminating noise enhancements as the noise figures of these amplifying devices are quite low [6,7]. The corresponding measured SNR at the output of this stage approximates to $18.6 \mathrm{~dB}$. In addition, the filtering circuit corresponds to a $2.45 \mathrm{GHz}$ bandpass filter that decreases significantly the level of the signals that are out of the frequency range of interest. For further analysis, this amplifying and filtering circuitry was investigated in terms of the S-parameters. Figure 3 shows the corresponding S21 parameter measurements. These results were provided by a Vector Network Analyzer and indicate the non-linear behavior of the current circuitry, giving a measure of unwanted phase and amplitude distortion that may be occured.

It is obvious that the offering power gain approximates to $42 \mathrm{~dB}$ for any value of the input (received) power. The corresponding small variations are quite negligible. Moreover, the AM to PM conversion is close to $0.2 \mathrm{deg} / \mathrm{dB}$ for the mean value of the input power $(-62.5 \mathrm{dBm})$. The reverse transmission coefficient is also a significant parameter that corresponds to the isolation characteristics of the current circuitry. Figure 4 depicts the measured results.

These results introduce $\mathrm{a}-57 \mathrm{~dB}$ isolation value that provides quite effective performance on the amplifying and filtering circuitry. Furthermore, Figure 5 represents the input return loss.

From these measurements, it is obvious that a quite small amount of the input power is reflected. This fact is
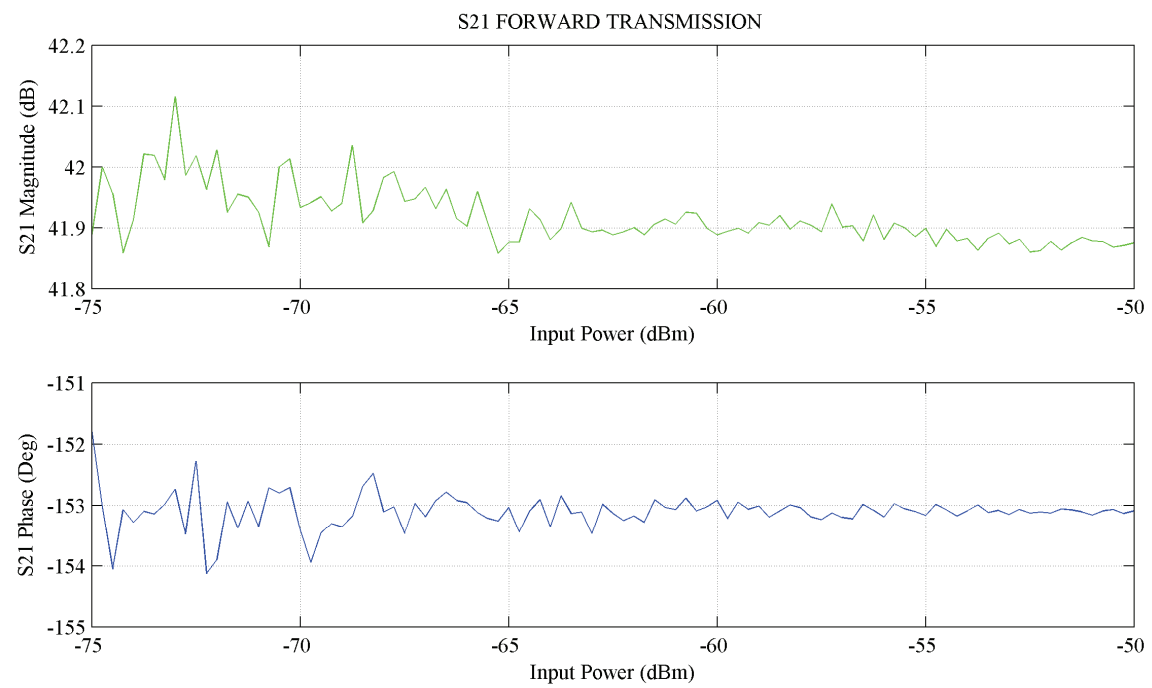

Figure 3. Transmission Gain of the first stage circuitry.

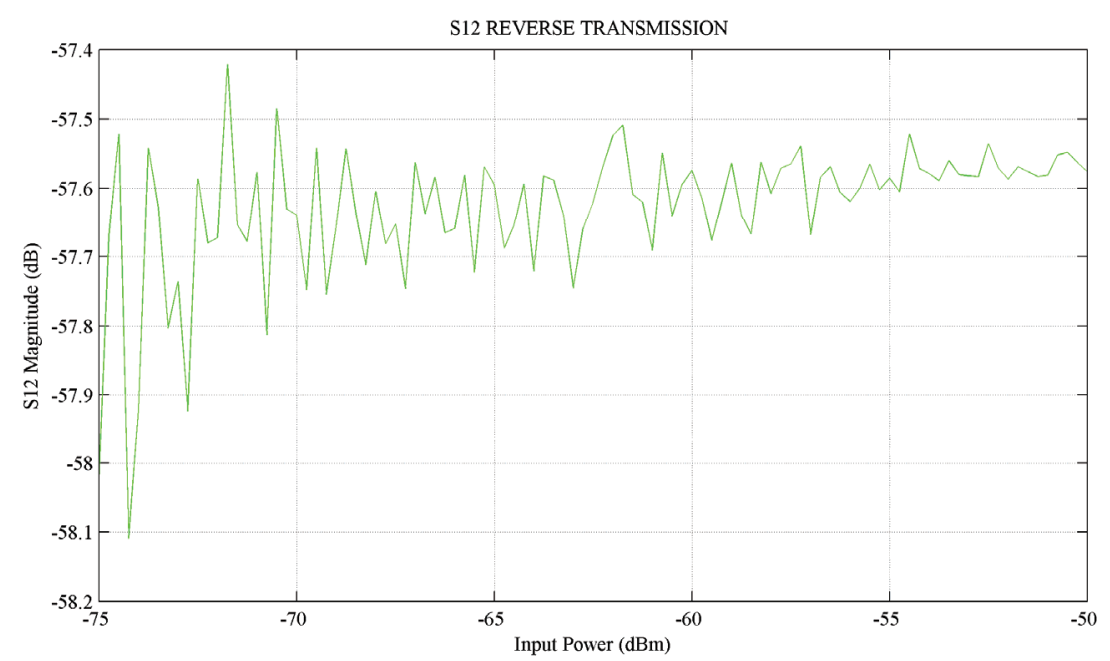

Figure 4. Isolation of the first stage circuitry. 


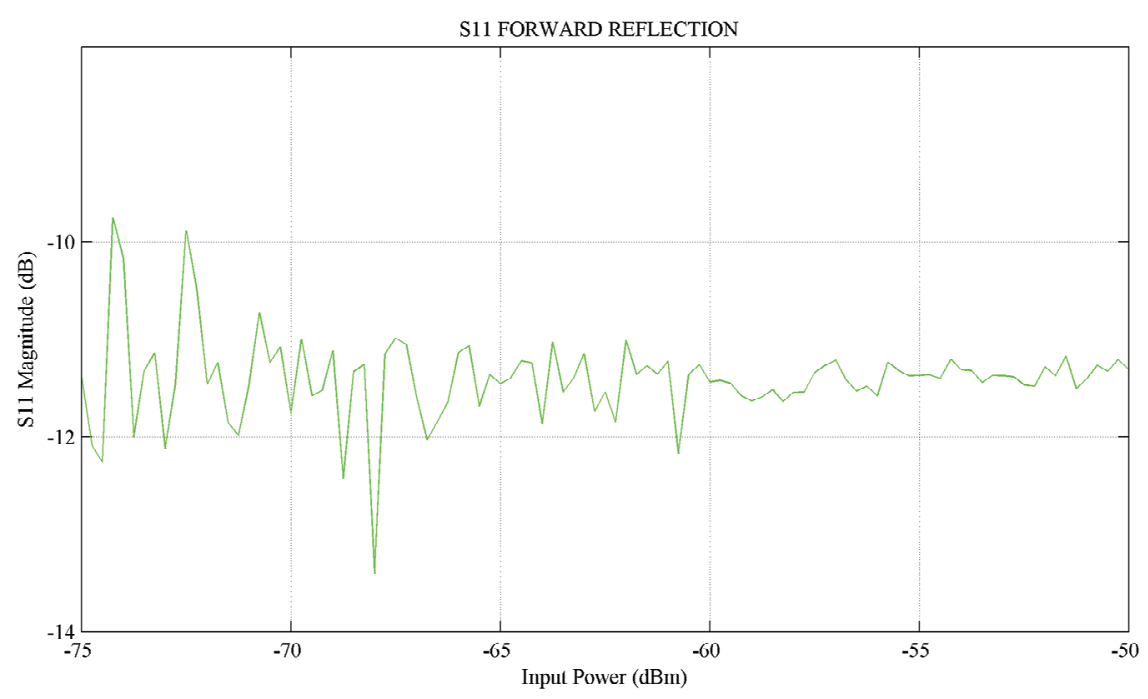

Figure 5. Input Return Loss of the first stage circuitry.

provided by the $-11 \mathrm{~dB}$ return loss value on the dynamic input power range of each receiver unit.

These characteristics indicate that the received RF modulated signal is prepared to drive the corresponding quadrature demodulator input at each of the four identical, albeit independent units. Demodulation procedure necessitates four local-oscillator signals that are provided via by a 1-to-4 power divider by a frequency generator at the frequency range of $2.4 \mathrm{GHz}$. Besides, the demodulator circuits offer quite $69.5 \mathrm{~dB}$ gain control adjustment on the RF signal strength at their corresponding outputs. For this, a precision control circuit sets the linear-in-dB gain response to the gain control voltage. Furthermore, these demodulator integrated circuits employ polyphase filters to achieve high quadrature accuracy and amplitude balance over the entire operation frequency range [8]. Each of the units provides $I x$ and $Q x$ output signals that correspond to the RF input signal, where $x$ is an index which ranges from 1 to 4 .

Test experimental measurements of the proposed implementation indicates constant amplitude and phase declinations presented on the signal outputs $(I x, Q x)$ due primarily to the different paths (transmission line lengths) from the LO source to the quadrature demodulator, as well as the demodulator inputs to the antenna array elements interface. These also include amplitude and phase errors from the coaxial lines that provide interconnection between RF inputs of the proposed receiver implementation with the elements of the antenna array [9]. These errors were measured and it is going to be taken into account in dynamic control on acquisition and data collection procedure.

\section{Multiple-Input Receiver Performance}

As noted above, the proposed multiple-input receiver implementation provides MIMO wireless communication and channel sounder applications in the frequency range of $2.4 \mathrm{GHz}$. For better analysis on the performance of this receiver device, a course of test measurements was made. For this purpose, signal generator platform was used to provide the RF inputs of the proposed implementation in order to simulate the signal reception of the corresponding antenna array. This equipment operates in transmit mode and provide four independent RF signals with several amplitudes, initial phases, frequencies and modulation modes. These signals are synchronized and drive the proposed receiver device for calibration and initialization purposes. Furthermore, a digital oscilloscope was also used to collect and store the $I x$ and $Q x$ output signals, for further analysis.

At first, signal amplitude variations were investigated by an appropriate local-oscillator and RF input signaling. Each receiver unit was fed by a single-tone signal at frequency $f_{R F}$ that differs from the LO signal frequency $f_{L O}$ by the parameter $d f$. The corresponding value ranges from $2 \mathrm{kHz}$ to $10 \mathrm{kHz}$, with step $2 \mathrm{kHz}$, providing a single tone IF signal at the corresponding $I x$ and $Q x$ output at frequency $d f$. Collection and acquisition of them were achieved via the digital oscilloscope equipment.

In addition, voltage gain control unit supports power gain adjustments on the RF signal at each demodulator input. A constant mean level was chosen at the corresponding dynamic range. The measured results are depicted in Figures 6-9 for each receiver unit, respectively. In these figures, the corresponding incoming RF power ranges from $-80 \mathrm{dBm}$ to $-52 \mathrm{dBm}$.

From these figures, it is obvious that the signal strength of the output $I x$ is quite constant at the frequency range of $10 \mathrm{kHz}$ for each value of $x$ parameter. Besides, the quadrature nature of the current demodulator ensures that the signal strength of the output $Q x$ var- 


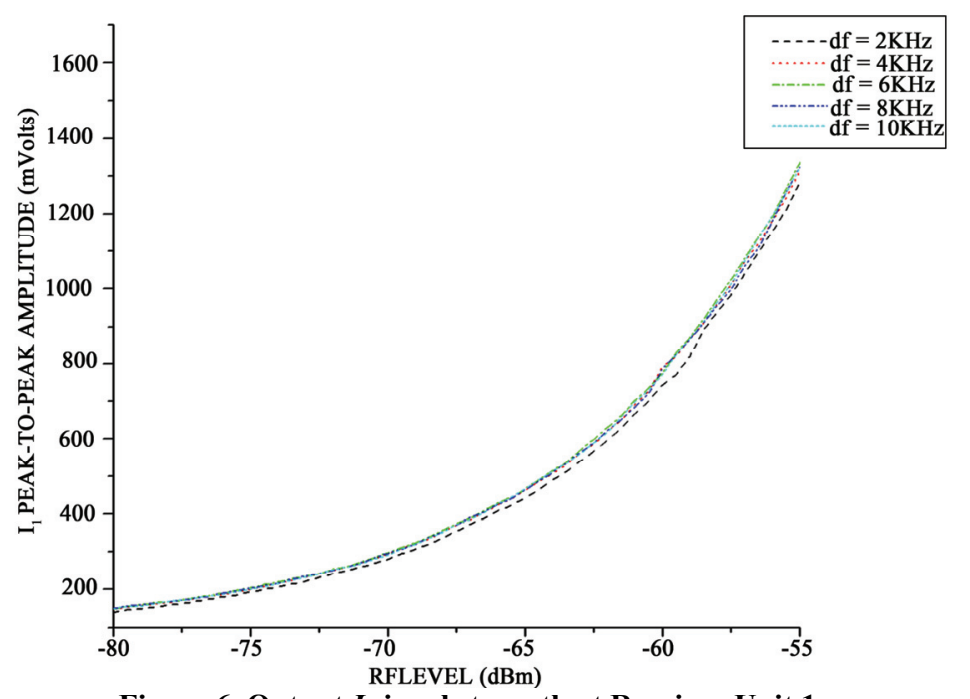

Figure 6. Output $I$ signal strength at Receiver Unit 1.

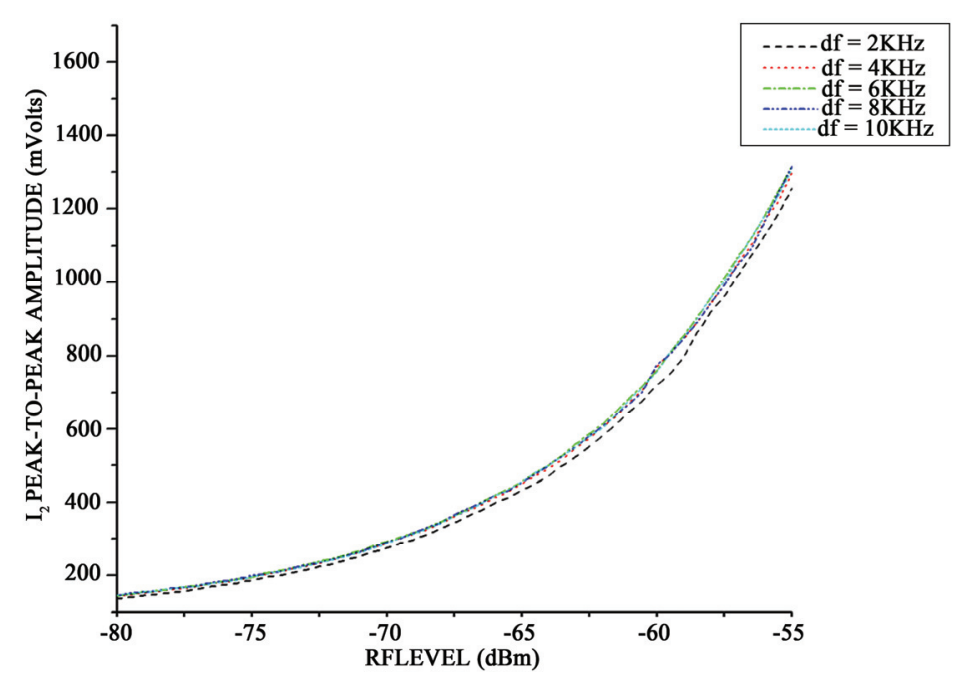

Figure 7. Output $I$ signal strength at Receiver Unit 2.

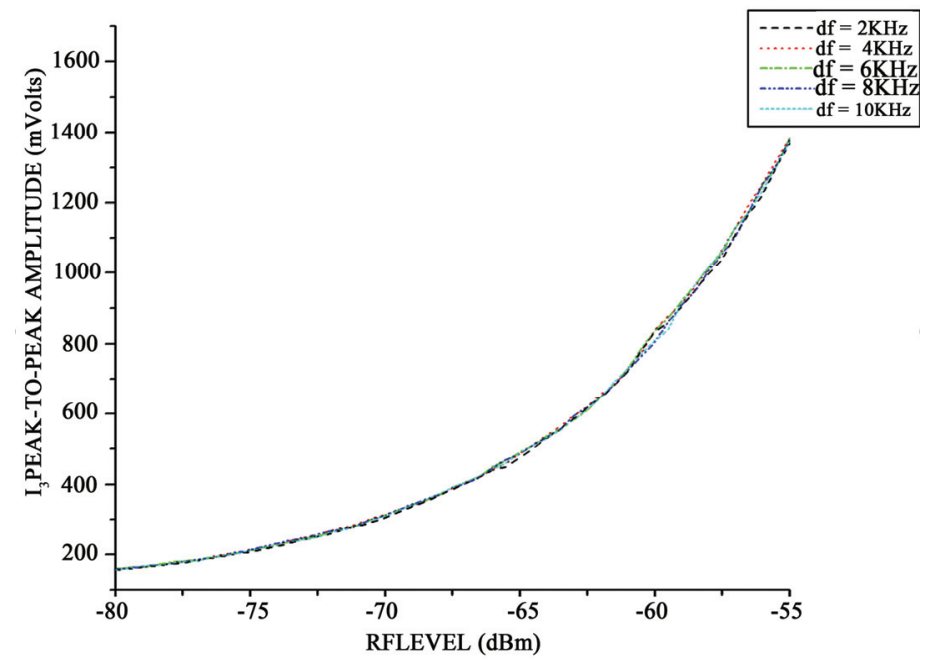

Figure 8. Output $I$ signal strength at Receiver Unit 3. 


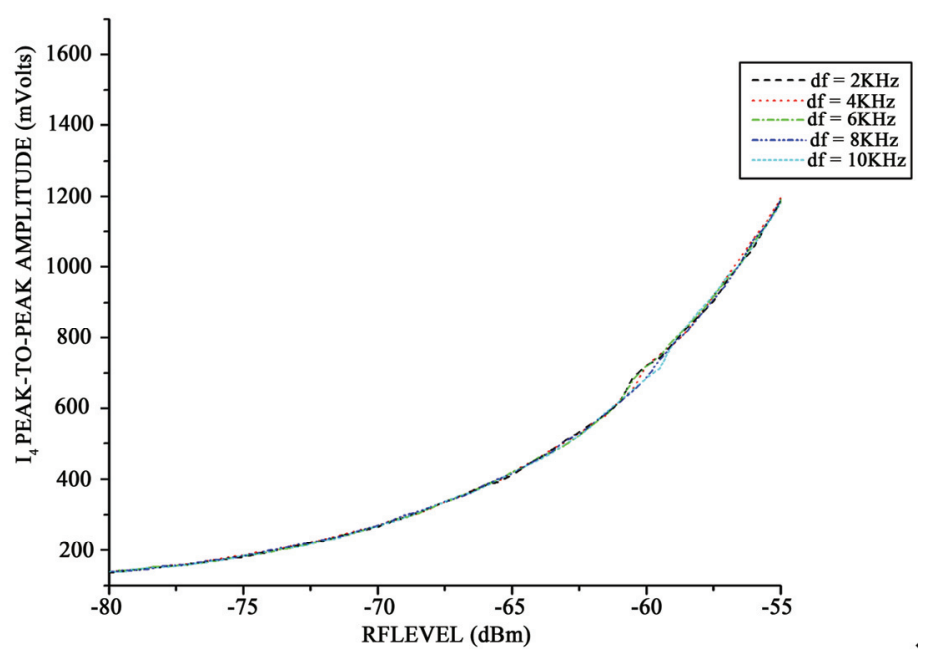

Figure 9. Output $I$ signal strength at Receiver Unit 4.

ies with the same way as the corresponding $I x$. There is only a 90 degrees phase shift amongst each pair $I x$ and $Q x$ at each receiver unit. As these curves have quite identical forms at the frequency range of $10 \mathrm{kHz}$, we chose a frequency point of $6 \mathrm{kHz}$ as the quite mean value on this bandwidth and we calculated the mathematical expressions that associates the output signal strengths with the RF received signal levels. Figures 10-13 depict these variations at each receiver unit, in order to provide the corresponding mathematical calculations. The corresponding results are included in Table $\mathbf{1}$.

These mathematical calculations introduce an effective way to define the received signal power in $\mathrm{dBm}$, when the value of $I x$ signal amplitude, expressed in voltage peak-to-peak, is obtained at each of the receiver units.

Furthermore, using the RF equipment that feed the proposed four channel receiver implementation, a course of calibration steps was made in order to investigate the phase difference on the receiving RF single tone signals. As mentioned above, these phase declinations are provided by the current receiver structure. For this, we used a pair of calibrated coaxial lines to connect the receiver RF inputs with the RF generator equipment. Two of the receiver units were fed by a single tone RF signal at frequency range of $2.4 \mathrm{GHz}\left(f_{R F}\right)$, simultaneously. In each case, the third receiver unit was used as reference. The corresponding LO inputs were also fed by a single tone signal at frequency $f_{L O}=f_{R F}+d f$, where $d f$ ranges from $1 \mathrm{kHz}$ to $10 \mathrm{kHz}$ with step $1 \mathrm{kHz}$. With this measured platform, we stored the phase difference $d p h i$ between the $I x$ output signals for several $d f$ values by a digital oscilloscope acquisition equipment. The corresponding results are presented in Figure 14.

As mentioned above, these $d p h i$ phase difference variations correspond to the frequency range of $2.4 \mathrm{GHz}$ with $10 \mathrm{kHz}$ bandwidth. The forms of these curves indicate that the parameter $d p h i$ is quite constant at this frequency range for each receiver unit. Moreover, receiver units 2 and 4 introduce quite identical phase shifts (24 degrees) with respect to the reference receiver unit 3 . Instead, the receiver unit 1 provides phase shifting on the order of 60 degrees. These declinations on phase shift values are caused by transmission line differences on the PCB layout and by declinations on linearity at the particular amplifying, filtering and demodulator circuitry.

In order to investigate the performance of the proposed receiver architecture on full MIMO channel characterization, we used this device with an appropriate four-element antenna array. In particular, these experimental results were provided by a single-element antenna and a single-channel RF platform at the transmitter end, as well as the four element antenna array and the proposed receiver implementation at the receiver end. Figures 15 and 16 depict the $I x$ output signals for two azimuth angle orientations of the antenna array at the receiver. These angles were 0 and 180 degrees and correspond to $\mathrm{A}$ and $\mathrm{B}$ antenna array orientations, respect-

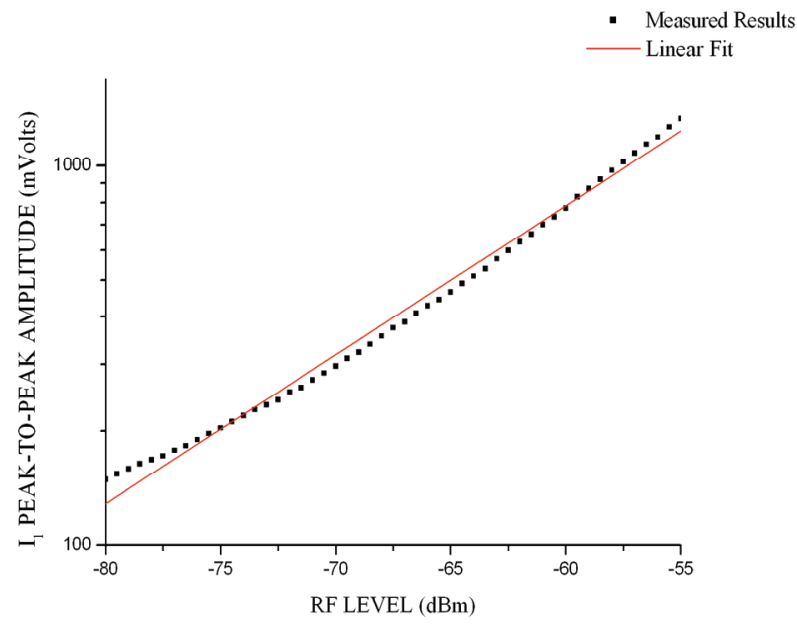

Figure 10. Linear fitting of the Output $I$ signal strength at Receiver Unit 1 for $d f=6 \mathrm{kHz}$. 


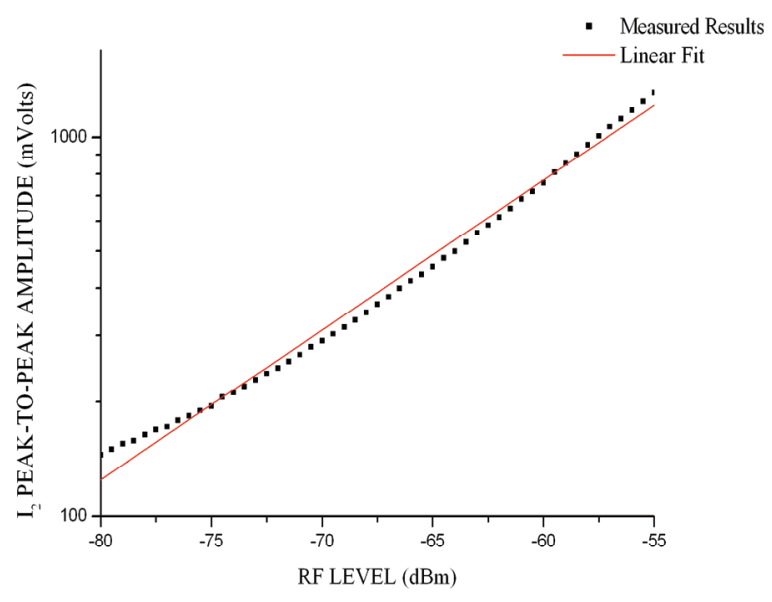

Figure 11. Linear fitting of the output $I$ signal strength at receiver unit 2 for $d f=6 \mathrm{kHz}$.

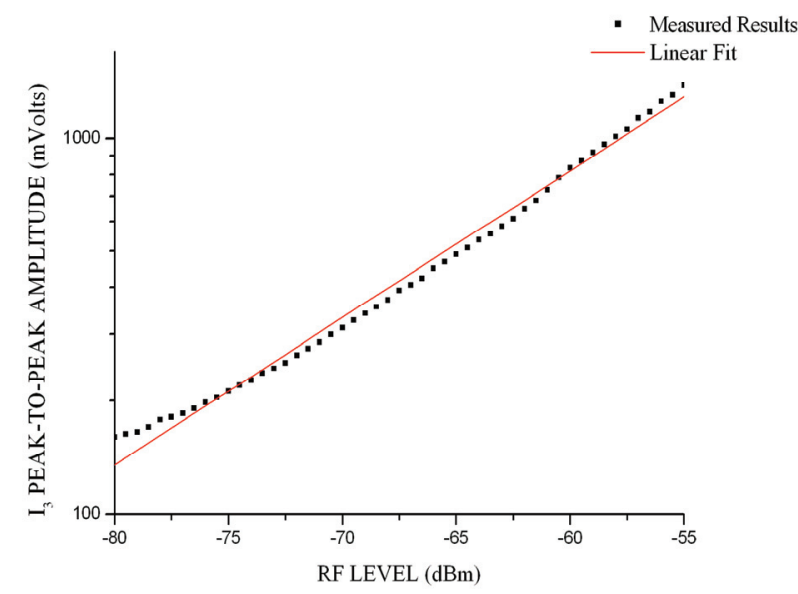

Figure 12. Linear fitting of the output $I$ signal strength at receiver unit 3 for $d f=6 \mathrm{kHz}$.

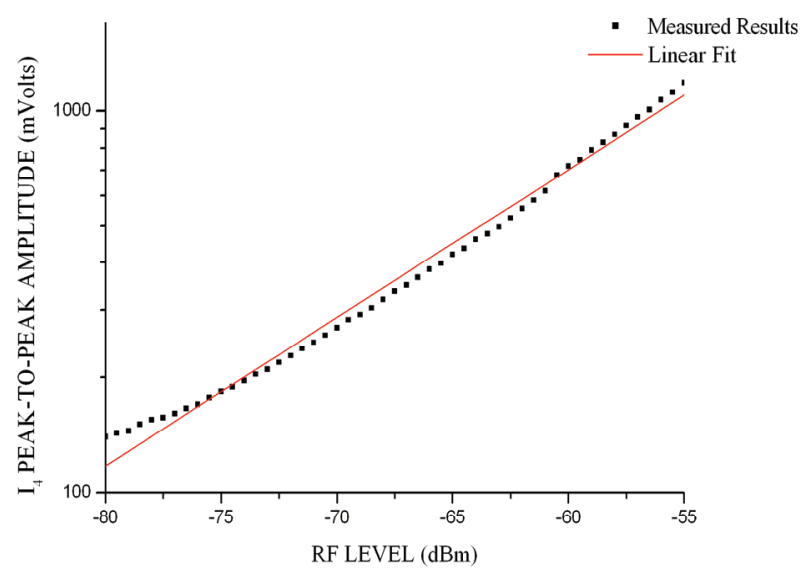

Figure 13. Linear fitting of the output $I$ signal strength at Receiver Unit 4 for $d f=6 \mathrm{kHz}$.

tively. In each case, the locations of the transmitter and receiver antenna, as well as the radio propagation environment were constant.
Table 1. Linear fit calculated results.

\begin{tabular}{|c|c|c|c|c|}
\hline \multicolumn{5}{|c|}{ Expression $\mathbf{Y}=\mathbf{A}+\mathbf{B} * \mathbf{X}$} \\
\hline $\begin{array}{c}\text { Receiver } \\
\text { unit }\end{array}$ & $\mathbf{A}$ & $\begin{array}{c}\text { Error of } \\
\text { A }\end{array}$ & B & $\begin{array}{c}\text { Error of } \\
\text { B }\end{array}$ \\
\hline First & 5.25 & 0.03 & 0.0393 & 0.0005 \\
\hline Second & 5.26 & 0.03 & 0.0396 & 0.0005 \\
\hline Third & 5.26 & 0.03 & 0.0392 & 0.0005 \\
\hline Forth & 5.18 & 0.03 & 0.0389 & 0.0005 \\
\hline
\end{tabular}

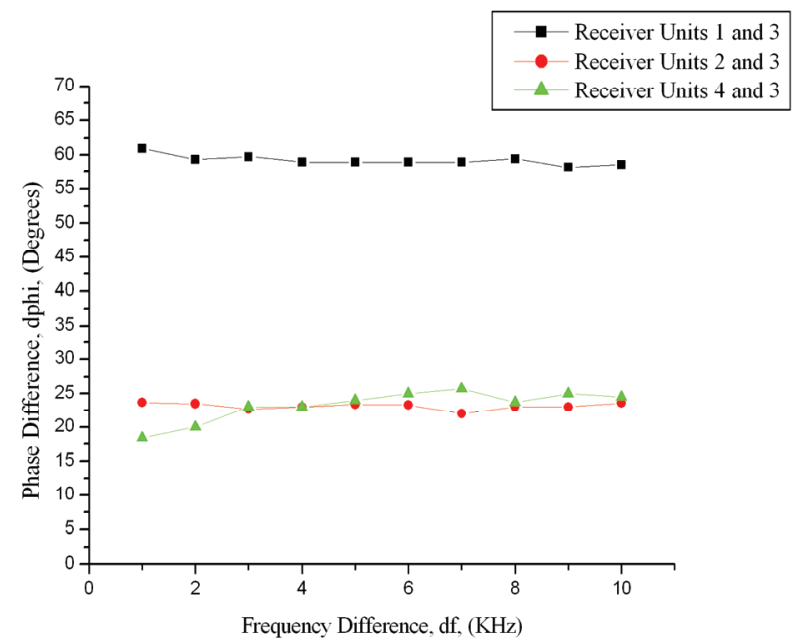

Figure 14. Phase declinations on the proposed receiver units.

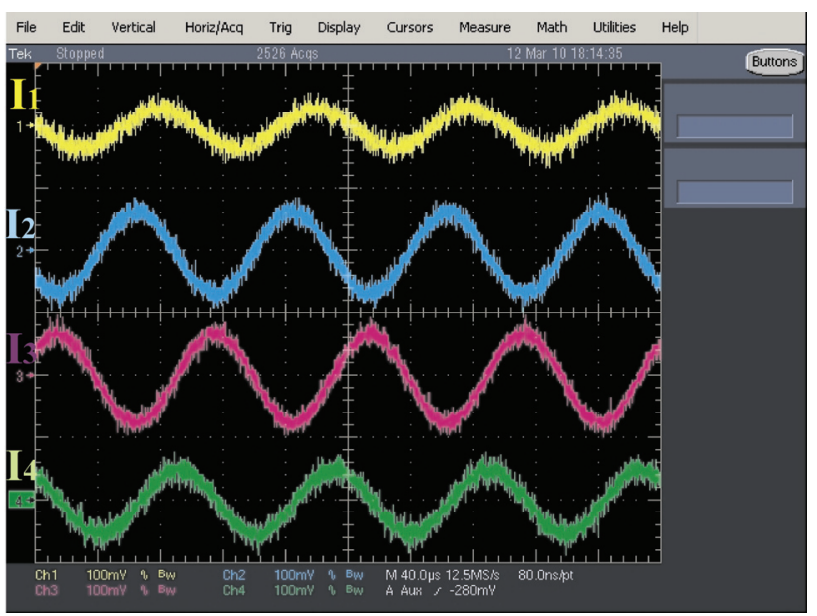

Figure 15. Output $I x$ signal strengths at Receiver for $d f=$ $10 \mathrm{kHz}$ and orientation $\mathrm{A}$.

From these representative measured results, it is obvious that the $I x$ output signals introduce amplitude and phase variations that correspond to the RF receiver signals. In fact, a single tone RF signal was transmitted and propagated via the multipath environment. As we used a four-element receiver antenna array, four copies of this RF signal were received. Each of them experiences different fading environment, decreasing the corresponding 


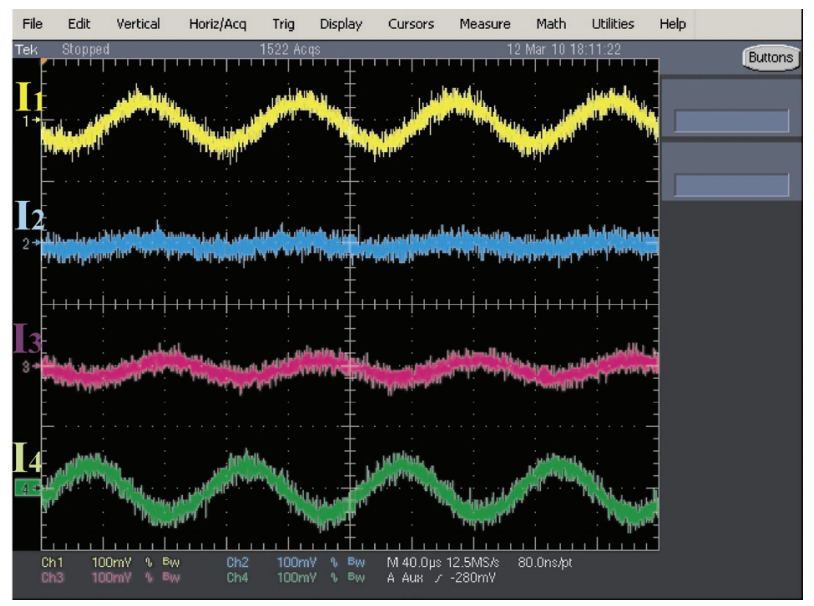

Figure 16. Output $I x$ signal strengths at Receiver for $d f=$ $10 \mathrm{kHz}$ and orientation $B$.

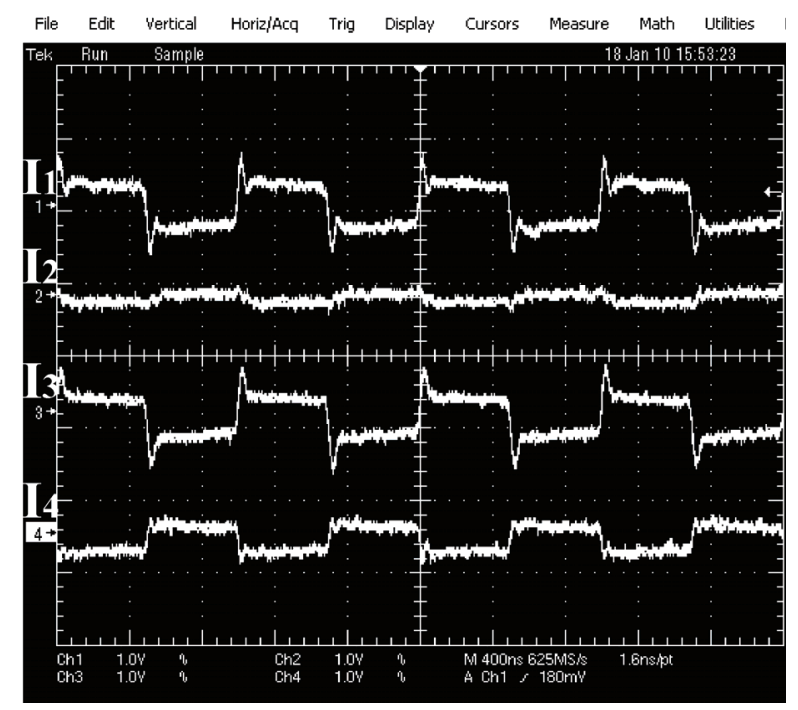

Figure 17. Data acquisition at BPSK modulation mode.

amplitude and changing its initial phase. Both these amplitude and phase variations are depicted in the corresponding $I x$ output signals of the proposed receiver device. These considerations indicate an interesting method of channel matrix calculations at several time snapshots of the radio propagation environment. As the proposed receiver implementation provides multiple-port applications and the $I x$ output signals are collected and recorded by a digital oscilloscope, simultaneously, we could exploit the experiment above, in order to study the full complex channel response between a multiple element antenna array at the transmitter and at the receiver ends, too. This procedure is known as MIMO channel sounder application.

Another issue is introduced by the quadrature demodulator devices that support the proposed receiver architecture. These integrated circuits provide direct demodulation, recovering the transmitted data stream. In par- ticular, we used the transmitter RF platform in order to provide a BPSK signal at the frequency range of $2.4 \mathrm{GHz}$. This modulated signal was transmitted by a single element antenna and received by a four element antenna array that was connected with the corresponding inputs of the proposed receiver structure. These results are included in Figure 17.

From this figure, it seems that the same data stream could be recovered in the receiver end at each of the four units in the proposed implementation. The corresponding $I x$ output signals depend on the common data codeword and the radio propagation environment at each channel between the transmitter and receiver ends. In particular, $I_{1}, I_{3}$ and $I_{4}$ output signals provide the data codeword in desirable form but the $I_{2}$ output signal has quite negligible amplitude. Besides, there is a time delay between $I_{4}$ and both $I_{1}$ and $I_{3}$. All these observations are provided by the corresponding channel propagation and multipath fading environment.

\section{Conclusions}

The design and construction of a multiple-input receiver, using state-of the art quadrature demodulation technique for MIMO wireless communication and channel sounder applications have been presented and investigated. The performance of the receiver in terms of frequency, phase and amplitude accuracy, as well as modulation mode and synchronization has been further discussed. Experimental measurements introduce the performance characteristics of the proposed multi-channel implementation according to the MIMO application requirements. In conclusion, the receiver design represents a versatile and efficient implementation for modern wireless applications. This design and an appropriate antenna array structure provide a RF platform for MIMO communications and channel characterization applications.

\section{Acknowledgements}

This research project (PENED) is co-financed by E.U.European Social Fund (80\%) and the Greek Ministry of Development-GSRT (20\%).

\section{References}

[1] H. Winters, "On the Capacity of Radio Communications Systems with Diversity in a Rayleigh Fading Envroment," IEEE Journal on Selected Areas in Communications, Vol. SAC-5, No. 5, 1987, pp. 871-878.

[2] G. J. Foschini, "Layered Space-Time Architecture for Wireless Communications in a Fading Environment When Using Multiple Antennas," Bell Labs Technical Journal, Vol. 1, No. 2, 1996, pp. 41-59.

[3] G. J. Foschini and M. J. Gans, "On Limits of Wireless Communications in a Fading Environment When Using 
Multiple Antennas," Wireless Personal Communications, Vol. 6, No. 3, 1998, pp. 311-335.

[4] M. H. Ullah and A. U. Priantoro, "A Review on Multiplexing Schemes for MIMO Channel Sounding," International Journal of Computer Science and Netwotk Security, Vol. 9, No. 6, 2009, pp. 294-300.

[5] S. A. Charles, E. A. Ball, T. H. Whittaker and J. K. Pollard, “A 5.5 GHz Channel Sounder for Fixed Wireless
Channels," Communications IEE Proceedings, Vol. 150, No. 4, 2003, pp. 253-258.

[6] "Analog Devices Preliminary Datasheets of MAX2640."

[7] "Mini-Circuits Preliminary datasheets of ERA-5."

[8] "Maxim Preliminary Datasheets of AD8347."

[9] D. M. Pozar, "Microwave Engineering," Wiley, New York, 1998. 